\title{
TLR8 Agonist DN1508052
}

National Cancer Institute

\section{Source}

National Cancer Institute. TLR8 Agonist DN1508052. NCI Thesaurus. Code C160500.

A small molecule Toll-like receptor 8 (TLR8; CD288) agonist with potential

immunostimulating and antineoplastic activities. Upon subcutaneous administration,

TLR8 agonist DN1508052 binds to TLR8, which is expressed in myeloid dendritic cells

(mDCs), monocytes, and natural killer (NK) cells. This may lead to the activation of

nuclear factor NF-kappa-B, the release of proinflammatory cytokines and a Th1-

weighted anti-tumor immune response. Additionally, activation of TLR8 signaling in

tumor cells may block the induction and reverse the suppression of senescent naive and

tumor-specific T-cells, resulting in enhanced anti-tumor immunity. TLR8, like other TLRs,

recognizes pathogen-associated molecular patterns (PAMPs) and plays a key role in

innate and adaptive immunity. 